\title{
Sistema sócio ecológico de uso dos recursos florestais por comunidades na Região de Santarém, Pará
}

As comunidades amazônicas, sejam ribeirinhas ou assentadas, ainda têm muita dificuldade em se desenvolver de forma sustentável. Este artigo buscou identificar se o Indicador de Desenvolvimento Econômico e Social (IDES), nas unidades de ocupação em estudo, está condicionado ao uso sustentável dos recursos florestais, foi utilizada a Análise Qualitativa Comparativa, com apoio da lógica de conjuntos difusos. Os resultados indicaram que a condição causal ideal era sistema de recurso aliado a sistema de governança para que o IDES pudesse ocorrer de forma satisfatória. Concluímos que o uso sustentável dos recursos florestais proporciona melhoria socioeconômica para as comunidades, desde que o uso seja realizado se respeitando as leis ambientais e as normas da comunidade.

Palavras-chave: Desenvolvimento Sustentável; Recursos Florestais; Uso da Terra; Fuzzy Set; Amazônia.

\section{Socio-ecological system of forest resource use by communities in the Santarém Region, Pará}

Amazonian communities, whether riverside or settled, still have great difficulty in developing themselves in a sustainable way. This article sought to identify if the Indicator of Economic and Social Development (IDES), in the occupational units under study, is conditioned to the sustainable use of forest resources, we used the Comparative Qualitative Analysis, with the support of the fuzzy sets logic. The results indicated that the ideal causal condition was a system of resource allied to a governance system so that IDES could occur in a satisfactory way. We conclude that the sustainable use of forest resources provides socioeconomic improvement for communities, provided that the use is made in compliance with environmental laws and community norms.

Keywords: Sustainable Development; Forest Resources; Land Use; Fuzzy Set; Amazon.

Topic: Área Temática

Reviewed anonymously in the process of blind peer.
Received: 10/08/2019

Approved: 28/09/2019
Everton Cristo de Almeida (id

Universidade Federal do Oeste do Pará, Brasil

http://lattes.cnpq.br/1713587609774692

http://orcid.org/0000-0002-3039-5440

evertonselva@yahoo.com.br

João Ricardo Vasconcellos Gama (1)

Universidade Federal do Oeste do Pará, Brasil

http://lattes.cnpq.br/9058536716453750

http://orcid.org/0000-0002-3629-3437

irvgama@gmail.com
Referencing this:

ALMEIDA, E. C.; GAMA, J. R. V.. Sistema sócio ecológico de uso dos recursos florestais por comunidades na Região de Santarém, Pará. Revista Ibero Americana de Ciências Ambientais, v.10, n.5, p.231242, 2019. DOI: http://doi.org/10.6008/CBPC2179$\underline{6858.2019 .005 .0020}$ 


\section{INTRODUÇÃO}

O desenvolvimento socioeconômico na Amazônia, mais especificamente no oeste paraense, teve seu auge em meio aos ciclos econômicos principais, com a comercialização das drogas do sertão, passando pela borracha, ouro, madeira, gado e soja. Esse avanço econômico só foi possível devido a expansão territorial dos grandes projetos de ocupação da Amazônia nas décadas de 60, 70 e 80 (OLIVEIRA et al., 2005).

Ao passo em que tudo isso acontecia na Amazônia, em Estocolmo - na Conferência das Nações Unidas de 1972, os assuntos sobre meio ambiente já eram discutidos em escala global, onde surgiu o termo 'ecodesenvolvimento' e em 1987 foi publicado o relatório 'Nosso Futuro Comum' ou 'relatório de Bruntland', apresentando ao mundo o conceito de desenvolvimento sustentável: satisfação das necessidades atuais, sem comprometer as fontes de recursos para as gerações futuras (NATIONS, 1987; MORAES, 2009).

Compondo a política e tentando resolver os conflitos de ocupação da Amazônia, implementou-se os Assentamentos e Unidades de Conservação de Uso Sustentável, foi uma estratégia adotada pensando, no caso dos assentamentos, na colonização e desenvolvimento regional, para cumprimento constitucional da função social da propriedade (BERGAMASCO, 1997; ROSSETO et al., 2003), e, no caso das Unidades de Conservação de Uso Sustentável a estratégia, além da conservação da biodiversidade foi o empoderamento dos recursos por parte das comunidades que habitam o local.

As pessoas que vivem nessas áreas praticam um sistema sócio ecológico muito complexo, que envolve dimensões bem distintas, mas que podem ser estudadas de forma conjunta por meio de Análise Qualitativa Comparativa, que tem como principal objetivo a identificação das condições necessárias e suficientes para que um determinado fenômeno ocorra (RAGIN, 2007).

A partir desse entendimento fez-se a pergunta: qual será o nível de utilização dos recursos florestais por estas comunidades?. Considerou-se a hipótese de que há uma relação harmônica entre o uso dos produtos florestais e a situação socioeconômica das comunidades. O presente artigo buscou identificar se $o$ Indicador de Desenvolvimento Econômico e Social nas unidades de ocupação em estudo, submetidos ao arranjo institucional local e governamental, está condicionado necessariamente ao uso sustentável dos recursos florestais.

\section{MATERIAIS E MÉTODOS}

A metodologia aplicada para a investigação da estrutura do Sistema Sócio ecológico (SSE) do uso dos recursos florestais nas diferentes unidades de ocupação na região de Santarém foi dividida em visitas às comunidades da margem direita do rio Maró-Arapiuns na RESEX Tapajós-Arapiuns e no Projeto de Assentamento Mojú I e II (Mapa 1). As comunidades ribeirinhas situadas na margem direita do rio MaróArapiuns, na RESEX Tapajós-Arapiuns, têm sua matriz populacional originária da própria localidade, sendo consideradas comunidades ou povos tradicionais. No Projeto de Assentamento Mojú le II as comunidades são compostas por colonos de diversas localidades do estado do Pará e de outros estados.

A temperatura do ar é sempre elevada, com média anual de $25,6^{\circ} \mathrm{C}+-9^{\circ}$, umidade relativa, com 
valores acima de $80 \%$ em quase todos os meses do ano, pluviosidade se aproxima dos $2.000 \mathrm{~mm}$ anuais, com certa irregularidade, durante todo o ano. As estações chuvosas coincidem com os meses de dezembro a junho e as menos chuvosas, com os meses de julho a novembro, tipo climático da região é o Ami, que se traduz como um clima, cuja média mensal de temperatura mínima é superior a 18 ำ tem uma estação seca de pequena duração e amplitude térmica inferior a 5 ํC entre as médias do mês mais quente e do mês menos quente (PACHECO et al., 2011). A vegetação que predomina é do tipo floresta ombrófila densa latifoliada comum das regiões tropicais, caracterizada por ser uma mata pesada e mista que pode se apresentar com cobertura florestal de maneira uniforme ou presença de árvores emergentes. Em menor proporção, dependendo da região geomorfológica, aparecem, Savanas, Campinaranas, Refúgio Ecológico, Floresta de igapó, estando inundada permanentemente no ano e significativas áreas de vegetação secundária (capoeiras) (VENTURIERI et al., 2010).

Os solos dominantes são pertencentes às classes dos Argissolos e Latossolos da Formação Barreiras, ambos constituídos por material mineral, apresentando-se nas cores amareladas, amarelo-avermelhadas e avermelhadas, com boas propriedades físicas e baixa fertilidade natural. Podemos encontrar também solos alagáveis ou alagados do conjunto estratigráfico da Formação Alter do Chão, que é representado por arenitos brancos a róseos, médios a grossos, com intercalações conglomeráticas e bancos, com níveis argilosos centimétricos a métricos (SOUSA, 2009). Foram selecionadas para o estudo, comunidades de um projeto de assentamento e de uma unidade de conservação, pois entendemos que são umas das principais representações de ocupação humana de uso sustentável na Amazônia (COSTA, 2014).
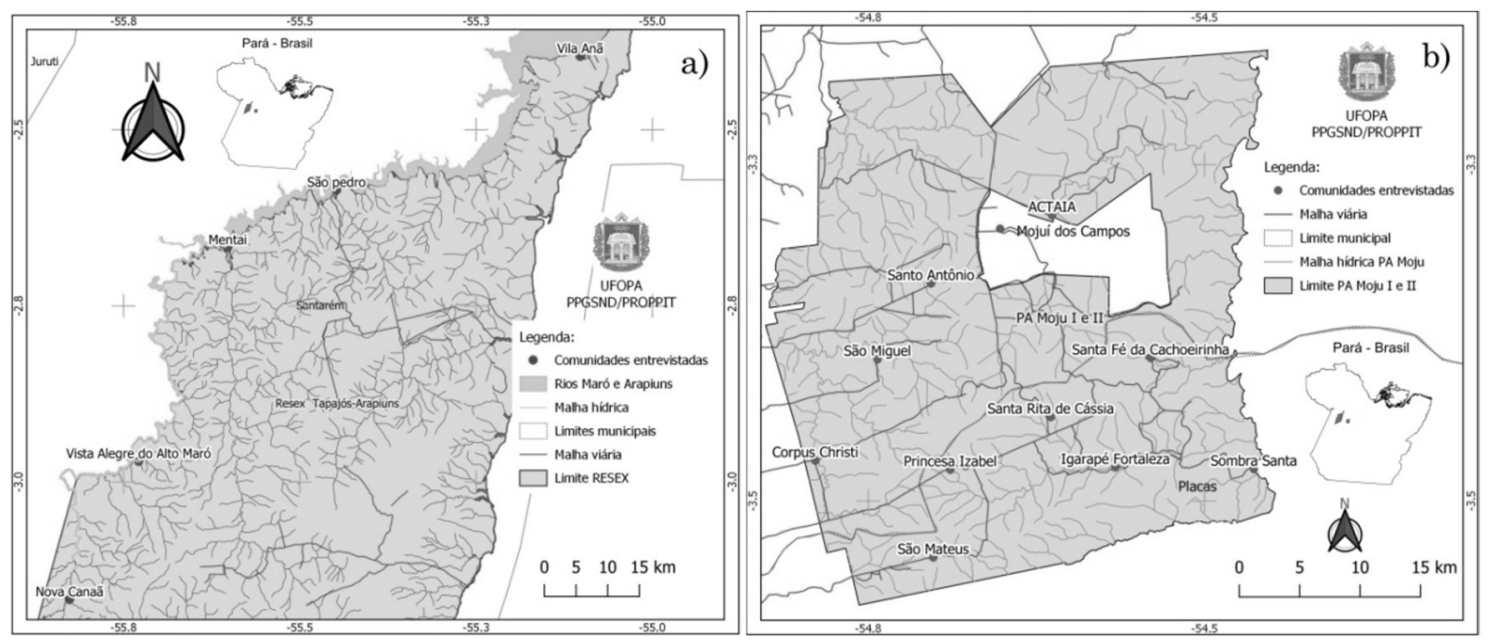

Mapa 1: Unidades de ocupação estudadas, Santarém - Pará. a) RESEX Tapajós-Arapiuns (comunidades da margem direita do rio Maró) e b) Comunidades do Projeto de Assentamento Mojú I e II.

As entrevistas foram realizadas com as lideranças de cada comunidade por indicação de especialistas de órgãos governamentais, não-governamentais e dos representantes das unidades de ocupação (PA Moju I e II e RESEX Tapajós-Arapiuns), que desenvolvem trabalhos de assistência técnica e que conhecem essas localidades. Os questionários aplicados foram estruturados conforme Khan (2002) e Leslie et al. (2015). O questionário foi composto de cinco dimensões e vinte e duas variáveis, como indicadoras, possuindo cada uma um peso específico (Tabela 1). 
Tabela 1: Variáveis utilizadas para análise dos Sistemas Sócio ecológicos nas comunidades do rio Maró e do PA Moju I e II, Santarém - Pará.

\begin{tabular}{|c|c|}
\hline Dimensões e variáveis & Pesos \\
\hline Dimensão 1 - Contexto Social, Econômico e Político (CS) & 1,00 \\
\hline CS1 - Desenvolvimento Econômico e Social & 0,30 \\
\hline CS2 - Tendências demográficas & 0,05 \\
\hline CS3 - Estabilidade política & 0,15 \\
\hline CS4 - Políticas públicas nas unidades de ocupação & 0,05 \\
\hline CS5 - Acesso aos mercados & 0,15 \\
\hline CS6 - Meios de comunicação e informação & 0,15 \\
\hline CS7-Tecnologias & 0,15 \\
\hline Dimensão 2 - Sistema de Recurso (SR) & 1,00 \\
\hline SR1 - Sistemas de recursos locais & 0,20 \\
\hline SR2 - Tamanho do Sistema de Recurso & 0,20 \\
\hline SR3 - Infraestrutura & 0,10 \\
\hline SR4 - Produtividade do sistema & 0,20 \\
\hline SR5 - Previsibilidade da dinâmica do sistema & 0,15 \\
\hline SR6 - Localização & 0,15 \\
\hline Dimensão 3 - Sistema de governança (SG) & 1,00 \\
\hline SG1 - Regras operacionais e coletivas de escolha & 0,50 \\
\hline SG2 - Privilégios territoriais de uso & 0,25 \\
\hline SG3 - Entidades que apoiam o uso do recurso florestal & 0,25 \\
\hline Dimensão 4 - Unidades de recursos (UR) & 1,00 \\
\hline UR1 - Diversidade de espécies florestais utilizadas & 0,50 \\
\hline UR2 - Valor econômico & 0,50 \\
\hline Dimensão 5 - Usuários ou Atores (U) & 1,00 \\
\hline U1 - Diversidade de atores relevantes & 0,30 \\
\hline U2 - Número de atores relevantes & 0,30 \\
\hline U3 - Migração & 0,20 \\
\hline U4-Isolamento & 0,20 \\
\hline
\end{tabular}

Os valores das variáveis da dimensão Contexto Social, Econômico e Político (CS), por representarem as condições básicas de qualidade de vida das comunidades, foram utilizados para calcular o Índice de Desenvolvimento Econômico e Social (IDES) (Expressão 1), para compor a variável de resposta, que juntamente com as variáveis independentes resultarão nas condições causais que levam ao cenário ótimo de desenvolvimento sustentável, por meio do uso dos recursos florestais nas comunidades em estudo.

Expressão 1: Expressão do Índice de Desenvolvimento Econômico e Social de cada comunidade.

Fonte: Adaptado de Khan (2002).

$$
\boldsymbol{I D E S}=\frac{1}{n} \sum_{j=1}^{n}\left[\sum_{i=1}^{m} E_{i j} \cdot P_{i j}\left(\sum_{i=1}^{m} E_{(\max ) i} \cdot P_{(\max ) i}\right)^{-1}\right]
$$

Onde: IDES = Índice de Desenvolvimento Econômico e Social; $\boldsymbol{P}_{i j}=$ Peso do $i$-ésimo indicador, alcançado pela $j$-ésima comunidade; $\boldsymbol{P}_{(\max ) i}=$ Peso máximo do $i$-ésimo indicador; $\boldsymbol{E}_{i j}=$ escore do $i$-ésimo indicador obtido pela $j$-ésima comunidade; $\boldsymbol{E}_{(\max ) i}=$ escore máximo do $i$-ésimo indicador; $i=1, \ldots, m$, número de indicadores; $j=1, \ldots, n$, número de comunidades.

Dessa forma, compomos o modelo que representa a condição causal que resulta em um cenário mais favorável para que haja o pleno desenvolvimento sustentável, utilizando os valores fuzzy das variáveis, que variam entre 0 e 1 (RAGIN, 2007). Os valores foram inseridos nos programas FsQCA V. 2.0 e Tosmana V. 1.52 (CRONQVIST, 2016), com a finalidade de encontrarmos as condições causais que resultam no cenário de pleno desenvolvimento sustentável, por meio da Análise Qualitativa Comparativa, utilizando a lógica dos conjuntos fuzzy.

Para testar se as variáveis apresentariam alguma relação linear, foram realizados os testes KMO 
(Kaiser-Meyer-Oclin) e o teste de esfericidade de Bartlett, como análise fatorial, a fim de reduzir as variáveis a somente um fator representativo (VICINI et al., 2005). Para ratificar a interpretação dos dados fuzzy, foi utilizada a análise de componentes principais (ACP) e análise de agrupamento, mas para tal, os valores fuzzy foram normalizados para serem processados no software PAST 3.0 (HAMMER et al., 2001) e no $R$ ( $R$ DEVELOPMENT CORE TEAM, 2011).

\section{RESULTADOS}

As análises relacionadas à Estrutura do Sistema Sócio ecológico do conjunto de comunidades nas duas regiões de ocupação de uso sustentável, revelaram que as condições causais refletem a necessidade do Sistema de Governança (SG), que representa as regras operacionais de gestão e escolhas locais, bem como todas as leis e regras governamentais vigentes, que têm influência sobre esses territórios e do Sistema de Recursos (SR), o qual representa toda riqueza em termos de diversidade fitofisionômica, bem como os sistemas de produção mistos (SAFs e ILPF), que são utilizados pelas comunidades, onde juntos, geraram um cenário de necessidade da variável $X$ para a variável $Y$, por meio da condição causal (SR*SG), com o valor de cobertura muito próximo de $1(0,992)$.

As combinações das condições geradas pelo algorítimo de Quine-McCluskey para encontrar as condições causais que levam ao cenário sustentável do Sistema Sócio ecológico, também são expressos no gráfico $X-Y$ dos conjuntos fuzzy, expressando que para o resultado esperado, grande parte dos valores estão abaixo da diagonal principal, apresentando uma relação de necessidade da condição causal apresentada, ou seja, o subconjunto (SR*SG) é maior ou igual ao resultado esperado para o Índice de Desenvolvimento Econômico e Social das comunidades (IDES) ou a combinação da condição causal é necessária para que a resposta do IDES ocorra de forma satisfatória, conforme o gráfico 1.

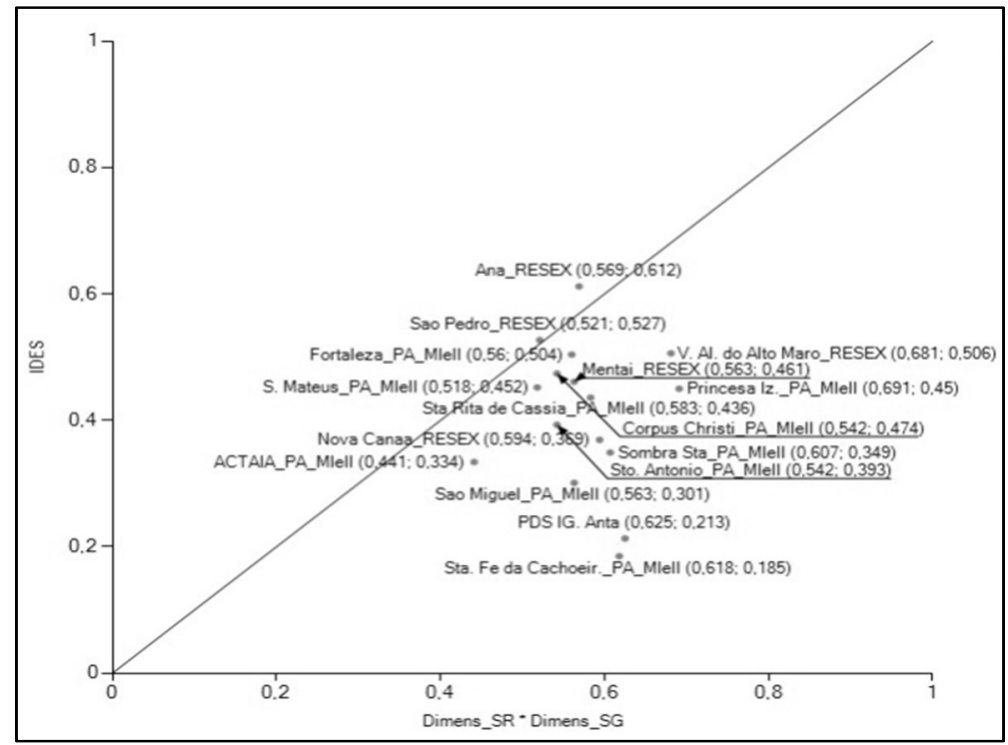

Gráfico 1: Gráfico $X-Y$ dos conjuntos fuzzy para o IDES $(X \geq Y)$.

A Análise Qualitativa Comparativa pelo Método Fuzzy set, a Análise de Agrupamento Hierárquico e a Análise de Componentes Principais mostraram resultados satisfatórios para detectar a combinação de 
fatores que explicam o funcionamento do quadro do Sistema Sócio ecológico das comunidades investigadas, levando-se em consideração as variáveis de primeira camada (CS, SR, SG, UR e U). A utilização do IDES das comunidades (Tabela 4) como variável de resposta para a operacionalização da FsQCA, na configuração do Sistema Sócio ecológico das comunidades, foi de extrema importância para entendermos quais as as condições causais suficientes ou necessárias para que haja desenvolvimento local.

Tabela 4: Informações socioeconômicas das comunidades do Rio Maro-Arapiuns e do PA Mojú I e II.

\begin{tabular}{|c|c|c|c|c|c|c|c|}
\hline $\mathbf{N}$ & Comunidades & $\begin{array}{l}\text { № de } \\
\text { famílias }\end{array}$ & IDES & $\begin{array}{c}\text { Renda } \\
\text { percapta (\$) }\end{array}$ & $\begin{array}{c}\text { Área } \\
\text { média } \\
\text { cultivada } \\
\text { (ha.ano-1) }\end{array}$ & $\begin{array}{c}\text { Distância } \\
\text { média da } \\
\text { UR (km) }\end{array}$ & $\begin{array}{c}\text { Valor médio } \\
\text { do PFM e NM } \\
\text { (R\$) }\end{array}$ \\
\hline 1 & Anã - RESEX Tapajós Arapiuns (Maró - Arapiuns) & 96 & 0,61 & 2,90 & 2 & 4 & 16,00 \\
\hline 2 & São Pedro - RESEX Tapajós Arapiuns (Maró - Arapiuns) & 100 & 0,53 & 1,90 & 2 & 13 & 100,00 \\
\hline 3 & Nova Canaã - RESEX Tapajós Arapiuns (Maró - Arapiuns) & 36 & 0,37 & 5,68 & 2 & 2 & 130,00 \\
\hline 4 & $\begin{array}{l}\text { Vista Alegre do Alto Maró - RESEX Tapajós Arapiuns } \\
\text { (Maró - Arapiuns) }\end{array}$ & 18 & 0,51 & 4,81 & 1 & 3 & 510,00 \\
\hline 5 & Mentai - RESEX Tapajós Arapiuns (Maró - Arapiuns) & 104 & 0,46 & 1,52 & 1 & 10 & 440,00 \\
\hline 6 & S. Mateus - PA Mojú I e II & 80 & 0,45 & 2,08 & 3 & 3,5 & 210,00 \\
\hline 7 & Princesa Izabel - PA Mojú I e II & 40 & 0,45 & 5,21 & 3 & 5 & 325,00 \\
\hline 8 & Sta Rita de Cássia - PA Mojú I e II & 56 & 0,44 & 3,13 & 1 & 1 & 60,00 \\
\hline 9 & Fortaleza - PA Mojú I e II & 35 & 0,50 & 5,95 & 3 & 1 & 233,00 \\
\hline 10 & Sombra Santa - PA Mojú I e II & 30 & 0,35 & 5,21 & 3 & 1,5 & 115,00 \\
\hline 11 & Sta. Fé da Cachoeirinha - PA Mojú I e II & 50 & 0,19 & 3,13 & 2 & 1 & 350,00 \\
\hline 12 & Santo Antônio - PA Mojú I e II & 54 & 0,39 & 2,89 & 1 & 1,5 & 150,00 \\
\hline 13 & Corpus Christi - PA Mojú I e II & 27 & 0,47 & 7,62 & 2,5 & 1 & 224,00 \\
\hline 14 & São Miguel - PA Mojú I e II & 37 & 0,30 & 4,81 & 1,5 & 3 & 400,00 \\
\hline 15 & ACTAIA - PA Mojú I e II & 32 & 0,33 & 6,25 & 1,25 & 1,5 & 166,70 \\
\hline 16 & PDS IG. Anta & 40 & 0,21 & 3,75 & 1,5 & 1 & 3,00 \\
\hline
\end{tabular}

A análise fatorial mostrou que existe correlação entre algumas variáveis do SSE, tendo resultados para o teste $\mathrm{KMO}=0,53$ e para o teste de esfericidade de Bartlett, com $p$-value $=0,02574$. Apesar da correlação média entre as variáveis, representado pelo teste $\mathrm{KMO}$, o teste de esfericidade de Bartlett rejeitou a hipótese nula $(\mathrm{HO})$ a um nível de significância $(\alpha=0,05)$, portanto implica dizer que há correlação entre algumas variáveis do SSE.

Procedeu-se a obtenção do coeficiente de correlação de Pearson para as variáveis investigadas, para sabermos quais os grupos de variáveis estariam mais correlacionados entre si e quais estariam menos correlacionados com variáveis de outros grupos, uma vez que o fator é representado por um grupo de variáveis identificadas pelas correlações observadas.

No gráfico 2, observamos a heterogeneidade entre as variáveis e entre as diferentes localidades do SSE, numa escala que varia entre 0 e 1, onde o maior valor indica que há gestão sustentável dos recursos florestais. Dentre as dimensões correlacionadas e analisadas via regressão linear simples, a relação Sistema de Recurso (SR) e Unidade de Recurso (UR) foi a mais alta e mais significativa, com $\alpha<0,05$.

As variáveis utilizadas para o cálculo das similaridades entre as comunidades, por meio da análise de agrupamento hierárquico com o método de Ward, foram todas as dimensões extraídas e padronizadas da estrutura do Sistema Sócio Ecológico, sendo elas o Sistema de Recursos (SR), Sistema de Governança (SG), Unidade de Recursos (UR), Usuários (U) e Contexto Social, econômico e Político (CS). Como resultado tivemos a linha de corte para três grupos definidos de acordo com a distância euclidiana, conforme o dendrograma 1. 


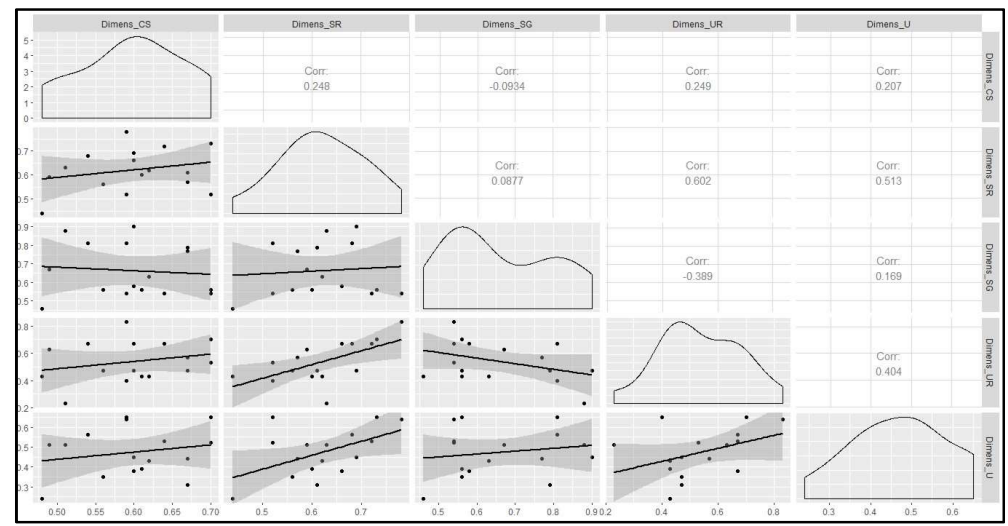

Gráfico 2: Gráfico de dispersão entre as variáveis de primeira camada do SSE nas diferentes localidades, suas correlações e a regressão linear simples com intervalo de confiança a 95\% e nível de significância $(\alpha=0,05)$.

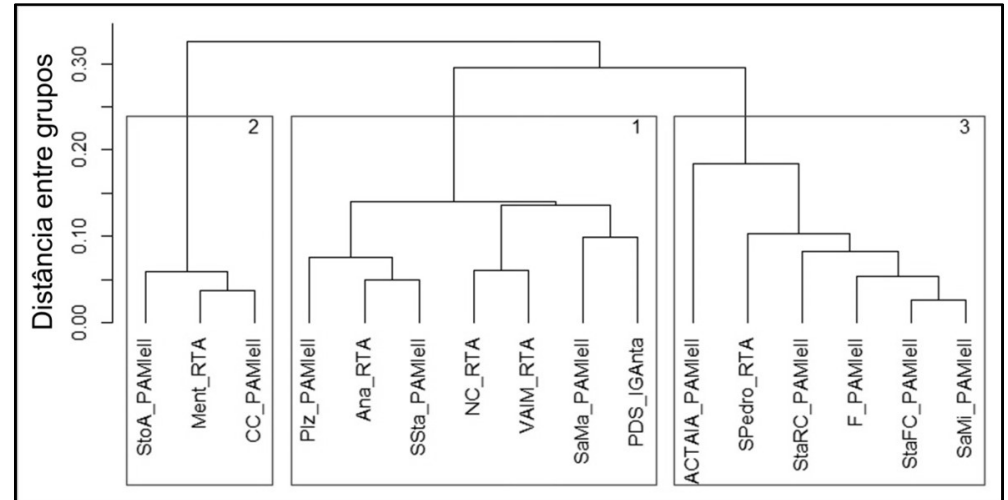

Dendrograma 1: Dendrograma obtido da análise de agrupamento hierárquico para as cinco variáveis do Sistema Sócio ecológico (CS, SR, SG, UR e U) pelo medo Ward.

O dendrograma mostra a similaridade das comunidades segundo as variáveis que completam o quadro do Sistema Sócio ecológico. As maiores similaridades estão entre as comunidades Santa Fé da Cachoeirinha (StaFC_PAMlell) e São Miguel (SaMi_PAMlell), Mentai (Ment_RTA) e Corpus Christi (CC_PAMlell) e, Anã (Ana_RTA) e Sombra Santa (SSta_PAMlell).

Para complementar a análise de agrupamento hierárquico, foi realizada a Análise de Componentes Principais (ACP) para identificar quais as variáveis mais representativas do Sistema Sócio ecológico para as diferentes comunidades pesquisadas. Na tabela 5 , as variáveis mais representativas para a componente principal 1 foram Sistema de Recurso (SR) e Unidade de recurso (UR), para a componente principal 2 foi o Sistema de Governança (SG) e CS para componente principal 3.

Tabela 5: Representatividade das variáveis nas componentes principais e a variação total cumulativa por componente principal.

\begin{tabular}{|l|l|l|l|}
\hline Dimensões & CP 1 & CP 2 & PC 3 \\
\hline CS & 0,33 & $-0,07$ & 0,94 \\
\hline SR & 0,57 & 0,18 & $-0,20$ \\
\hline SG & $-0,10$ & 0,84 & 0,06 \\
\hline UR & 0,57 & $-0,32$ & $-0,26$ \\
\hline U & 0,49 & 0,38 & $-0,08$ \\
\hline Cumulativo (\%) & $\mathbf{4 3 . 4}$ & $\mathbf{6 8 . 5}$ & $\mathbf{8 5 . 4 6}$ \\
\hline
\end{tabular}

No gráfico das cargas fatoriais (gráfico 3), podemos observar que associada à variável (UR) estão as comunidades Corpus Christi, Santo Antônio e Mentai, com os maiores autovalores na componente principal 
1. Para a variável (SG) estão associadas as comunidades Princesa Izabel, São Mateus e o PDS Igarapé do Anta, com maiores autovalores da componente principal 2. As comunidades Sombra Santa, Santa fé da Cachoeirinha, São Miguel, Igarapé da Fortaleza, São Pedro, Anã, Nova Canaã, Santa Rita de Cássia e, principalmente a comunidade ACTAIA, não estão associadas ás variáveis que compõem o Sistema Sócio ecológico, segundo a ACP.

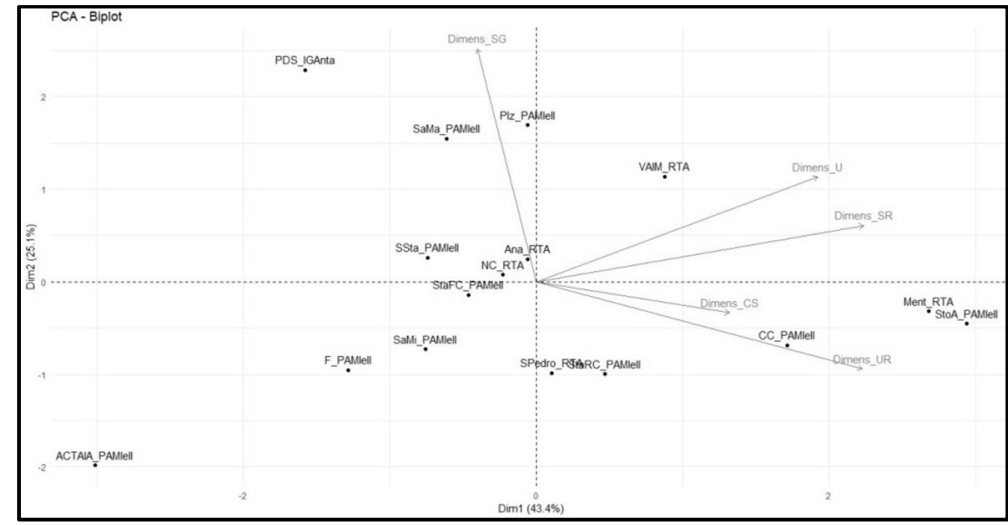

Gráfico 3: Gráfico das cargas fatoriais da ACP para as variáveis do SSE.

Por meio das informações cumulativas do percentual de explicação da variância total das componentes principais, observamos que não foi possível a redução das variáveis para um único fator, tendo que aceitar duas ou mais componentes principais para explicar a variância total. Identificamos que as variáveis UR, SR e SG, em conjunto, tem forte influência sobre o quadro de sustentabilidade associada ao Desenvolvimento, Econômico e Social (IDES), ou seja, o uso dos recursos florestais madeireiros e não madeireiros ( Unidades de Recursos - UR), bem como o estoque representado pelo (Sistema de Recurso Florestal - SR), tais como florestas primárias, secundárias e SAFs, todos sendo submetidos a um conjunto de regras locais e governamentais, geridas pelas comunidades (Sistema de Governança - SG) é capaz de organizar e viabilizar o planejamento e uso desse recurso, resultando na melhoria da qualidade de vida de algumas comunidades. Na tabela 6 , estão as espécies mais utilizadas pelas comunidades pesquisadas, tanto madeireiras como não madeireiras, localizadas em plantio (SAFs) ou adquiridas por meio do extrativismo.

Todas as espécies florestais da tabela 6 acima são as mais utilizadas e as mais importantes nas comunidades, muitas delas indispensáveis, sendo alguns produtos florestais madeireiros e não-madeireiros. Podemos destacar que as espécies florestais Itaúba, Jarana e Maçaranduba são as mais utilizadas e os mais importantes produtos madeireiros, já entre os não-madeireiros destacaram-se a Andiroba, Copaíba, Piquiá e Cumarú, onde mais da metade das comunidades entrevistadas fazem seu uso e sua comercialização.

Tabela 6: Frequência relativa das unidades de recursos florestais, classificados em produtos florestais madeireiros (PFM) e Não Madeireiros (PFNM), para as 16 comunidades entrevistadas.

\begin{tabular}{|l|l|l|l|}
\hline Nome Vulgar & Nome Científico & Freq. Relativa (\%) & Tipo \\
\hline Açaí & Euterpe oleracea Mart. & 43,8 & PFNM \\
\hline Acariquara & Minquartia guianensis Aubl. & 12,5 & PFM \\
\hline Andiroba & Carapa guianensis Aubl. & 75,0 & PFNM \\
\hline Angelim pedra & Hymenolobium petraeum Ducke & 6,3 & PFM \\
\hline Araraúba & Aspidosperma desmanthum Benth. ex Müll. Arg. & 12,5 & PFM \\
\hline Bacaba & Oenocarpus bacaba Mart. & 12,5 & PFNM \\
\hline Buriti & Mauritia flexuosa Mart. & 6,3 & PFNM \\
\hline
\end{tabular}




\begin{tabular}{|c|c|c|c|}
\hline Castanha do Pará & Bertholletia excelsa H.B.K. & 43,8 & PFNM \\
\hline Cedro & Cedrela fissilis Veli. & 25,0 & PFM \\
\hline Cipó titica & Heteropsis flexuosa (H.B.K.) G. S.Bunting & 25,0 & PFNM \\
\hline Copaíba & Copaifera langsdorffii Desf. & 68,8 & PFNM \\
\hline Cumaru & Dipteryx odorata (Aublet.) Willd. & 50,0 & PFM e NM \\
\hline Cupiuba & Goupia glabra Aubl. & 31,3 & PFM \\
\hline Fava tamboril & Enterolobium maximum Ducke. & 6,3 & PFM \\
\hline Ipê amarelo & Handroanthus serratifolius (Vahl) S.Grose & 18,8 & PFM \\
\hline Itaúba & Mezilaurus itauba (Meisn.) Taub. ex Mez. & 93,8 & PFM \\
\hline Jacitara & Desmoncus giganteus A.J.Hend. & 6,3 & PFNM \\
\hline Jarana & Lecythis jarana (Huber ex Ducke) A.C. Sm. & 50,0 & PFM \\
\hline Jatobá & Hymenaea courbaril L. & 12,5 & PFM \\
\hline Jucá & Caesalpinia ferrea Mart. Ex Tul.var. ferrea. & 6,3 & PFNM \\
\hline Maçaranduba & Manilkara huberi (Ducke) Standl. & 50,0 & PFM \\
\hline Muiracatiara & Astronium lecointei Ducke & 6,3 & PFM \\
\hline Muirapixuna & Cassia scleroxylon Ducke & 6,3 & PFM \\
\hline Piquiá & Caryocar villosum (Aubl.) Pers. & 68,8 & PFM e NM \\
\hline Pupunha & Bactris gasipaes Kunth & 12,5 & PFNM \\
\hline Quaruba & Vochysia maxima Ducke & 18,8 & PFM \\
\hline Sucuba & Himatanthus sucuuba (Spruce ex Müll. Arg.) & 12,5 & PFNM \\
\hline Tauari & Couratari guianensis. Aubl. & 6,3 & PFM \\
\hline Tucumã & Astrocaryum vulgare Mart. & 6,3 & PFNM \\
\hline Uxi & Endopleura uchi (Huber) Cuatrec & 18,8 & PFM e NM \\
\hline
\end{tabular}

\section{DISCUSSÃO}

As comunidades envolvidas na pesquisa têm perfis diferentes, com formação da estrutura social e econômica distintas, no entanto, quando se trata de uso dos recursos florestais elas possuem algumas semelhanças que puderam ser observadas por meio da Análise Qualitativa Comparativa, operacionalizada pelo método Fuzzy set.

A combinação das variáveis de primeira camada (SR) e (SG), tendo como a variável resposta o Índice de Desenvolvimento Econômico e Social (IDES), obteve valores de cobertura 0,992 e consistência 0,707, implicando em uma condição necessária para que o IDES possa ocorrer de forma satisfatória. Para a estrutura do Sistema Sócio ecológico, este resultado mostra que o cenário mais sustentável está relacionado com a forma como o recurso florestal é gerido, por meio das instituições (regras locais), bem como os procedimentos legais de licenciamento e regulação do uso dos recursos, representado pelo Sistema de Governança (SG), que contribuem para a manutenção do estoque, representado pelo Sistema de Recurso (SR). Estoque composto pelos recursos florestais madeireiros e não madeireiros, que contribuem na renda da maioria das famílias, juntamente com a produção da agricultura familiar. Resultados similares ocorreram com (LESLIE et al., 2015).

Para McGinnis et al. (2014) o Sistema Sócio ecológico é baseado na teoria do uso dos recursos comuns, que no nosso caso é representado pelos recursos florestais utilizados por diferentes usuários, nas suas diferentes formas, oriundos de diferentes sistemas de recursos. No entanto, refere-se também na capacidade de se restabelecer e permanecer para as próximas gerações, remetendo aos conceitos de resiliência e sustentabilidade dentro dos Sistemas Sócio ecológicos, descritos em profundidade por (ATHAYDE et al., 2016; BERKES et al., 2003; OLSSON et al., 2004; WALKER et al., 2004).

Apesar das comunidades estarem inseridas em diferentes unidades de ocupação territorial, com históricos distintos de desenvolvimento, comunidades do Projeto de Assentamento Mojú l e Il e da calha dos 
rios Maró-Arapiuns, na RESEX Tapajós-Arapiuns, apresentaram algumas semelhanças entre variáveis, isso pôde ser evidenciado por meio das análises fatoriais realizadas para as variáveis do SSE. A ACP não foi capaz de reduzir as variáveis a apenas um fator principal, o que nos fez optar pela escola das variáveis mais representativas na componente 1 e 2 . Coincidentemente as variáveis foram as mesmas indicadas peã FsQCA, Sistemas de Recursos, Sistema de Governança, além da variável Unidade de recurso. Isso confirma que essas variáveis tem grande peso para que haja desenvolvimento sustentável por meio do uso dos recursos florestais.

Entre as comunidades que tiveram maiores similaridades, destacamos Mentai (Rio Maro-Arapiuns) e Corpus Christi (PA Moju I e II). Essas comunidades apresentaram como variáveis latentes a Unidade de Recursos (UR) e Sistemas de Recursos (SR), que envolve o uso dos produtos florestais madeireiros e não madeireiros, bem como entendem a importância dos estoques florestais para as gerações futuras. A componente principal 2 demonstrou que outra variável pôde ser considerada na $A C P$, a que representa o Sistema de Governança (SG), que é uma variável composta pelas regras locais (instituições) e regras legais para uso, baseadas nos processos de licenciamento do uso dos recursos florestais nessas comunidades.

Nas entrevistas realizadas nas comunidades, ficou claro que o uso do recurso florestal não depende somente da disponibilidade do recurso e das regras locais que ordenam esse uso, mas também a presença de atores externos às comunidades, responsáveis por viabilizar as atividades mais rentáveis, como o Manejo Florestal Sustentável. Tais atores sociais são empresas madeireiras e empresas de consultoria florestal, responsáveis pelas atividades de Manejo. Em assentamentos o que há é uma iminente necessidade de se utilizar do recurso florestal madeireiro como forma de capitalização do possuidor do lote, com a finalidade de autofinanciar agricultura familiar. Sendo o Manejo realizado nos lotes de até 100 hectares, explorando 80\% desses lotes, que são aninhados em uma única unidade de produção anual (UPA). Nós entendemos que esta modalidade de Manejo não é sustentável, uma vez que o assentado fica impossibilitado de realizar um novo manejo até completar o ciclo de 30 anos, ficando mais vulnerável para a prática de retirada ilegal de madeira entre outros crimes ambientais praticados na Reserva Legal.

No PA Mojú I e II, as decisões sobre o manejo florestal sempre foram uma proposta ou convite realizado pelas comunidades, mas muitas vezes as empresas sondam as comunidades no intuito de conseguir desenvolver a atividade de diversas formas e isso pode indicar que a atividade será conduzida utilizando as regras das empresas madeireiras, onde o comunitário geralmente não participa das atividades, libera sua propriedade para exploração e muitas das vezes não acompanham qual foi a produtividade, vendendo a madeira em tora com valores que variam entre $R \$ 60,00$ e $R \$ 100,00$, muito inferiores quando a madeira é processada para venda.

No caso das comunidades da RESEX Tapajós-Arapiuns, mais especificamente da calha do MaróArapiuns, têm uma visão diferenciada do uso do recurso florestal. Apesar de saber que existe um ativo imobilizado na floresta primária, que faz parte dos limites das comunidades, as comunidades tradicionais pensam no modelo sustentável como uma oportunidade ímpar para as próximas gerações.

Portanto, o processo de uso do recurso florestal é realizada por meio de chamada pública, uma vez 
que existe uma concessionária, que é a pessoa jurídica responsável por representar os interesses das comunidades da RESEX Tapajós-Arapiuns, a Tapajoara. Dessa forma, o processo é conduzido sob a observação de um conselho consultivo e um grupo de trabalho que se dedica às discussões sobre a melhor forma das comunidades realizarem as atividades de Manejo Florestal, obedecendo a legislação vigente e visando sempre a sustentabilidade da atividade. Estudos em Unidades de Conservação com implementação do método de apoio à tomada de decisão na verificação da sustentabilidade, por meio da FsQCA, apoiando o Sistema Sócio ecológico, já foram realizados por (CAMPOS FILHO, 2004; ROSAS, 2012), onde esta ferramenta mostrou-se muito eficaz.

\section{CONCLUSÕES}

As comunidades usam de forma diferenciada o recurso florestal, as comunidades ribeirinhas são mais extrativistas, aproveitam melhor os produtos florestais madeireiros e não madeireiros oriundos de floresta nativa. E as comunidades assentadas usam com menor intensidade os produtos florestais e preferem plantar árvores com a certeza do retorno econômico, como andiroba para a produção de óleo, o cumaru, para venda de sementes e o piquiá, que serve como madeira, fruto ou extração de óleo. $\mathrm{O}$ uso dos recursos florestais e o sistema de governança são as dimensões que definem o nível de desenvolvimento socioeconômico das comunidades.

\section{REFERÊNCIAS}

ATHAYDE, S.; BERNASCONI, P.; BARTELS, W. L.; SELUCHINESK, R.; BUSCHBACHER, R.. Avaliação da resiliência socioecológica como ferramenta para a gestão da fronteira amazônica: experiências e reflexões. Sustentabilidade em Debate, v.7, n.2, p.14, 2016.

BERGAMASCO, S. M. P. P.. A realidade dos assentamentoos rurais por detrás dos números. Estudos Avançados, v.11, n.31, p.37-49, 1997.

BERKES, F.; COLDING, J.; FOLKE, C.. Navigating socialecological systems: building resilience for complexity and change. Cambridge: Cambridge University Press, 2003.

COSTA, F. S. . A dinâmica dos recursos comuns em Unidades de Conservação e Assentamentos Rurais no Amazonas: uma abordagem fuzzy set. Belém: Universidade Federal do Pará, 2014.

CRONQVIST, L.. Tosmana, V. 1.52. Trier: Germany University of Trier, 2016.

CAMPOS FILHO, P.. Método para apoio à decisão na verificação da sustentabilidade de uma unidade de conservação, usando lógica fuzzy. Tese (Doutorado em Engenharia de Produção) - Universidade Federal de Santa Catarina, Florianópolis, 2004.

HAMMER, $\varnothing . ;$ HARPER, D. A. T.; RYAN, P. D.. Past: paleontological statistics software package for education and data analysis. 2001.

KHAN, A. S.. Reforma Agrária Solidária, Assistência Técnica e
Desenvolvimento Rural no Estado do Ceará. Revista Econômica do Nordeste, v.33, n.3, p.593-614, 2002.

LESLIE, H. M.; BASURTO, X.; NENADOVIC, M.; SIEVANEN, L.; CAVANAUGH, K. C.. Operationalizing the social-ecological systems framework to assess sustainability. PNAS, v.112, n.19, p.5979-5984, 2015.

MCGINNIS, M. D.; OSTROM, E.. Social-ecological system framework: initial changes and continuing challenges. Ecology and Society, v.19, n.2, p.12, 2014.

MORAES, O. J.. Economia ambiental: instrumentos econômicos para o desenvolvimento sustentável. São Paulo: Centauro, 2009.

NATIONS, U.. Report of the World Comission on Enviroment and Development: Our Common Future. Estocolmo, 1987.

OLIVEIRA, A. U.; OLIVEIRA, B. C.; FEARNSIDE, P. M.; ARAGÃO, J.; ORRICO, R.; ROCHA, J.; FIGUEIREDO, W.; FILHO, A. C.; ABEX JR., J.; TORRES, M.. Amazônia revelada: os descaminhos ao longo da BR-163. Brasília: CNPQ, 2005.

OLSSON, P.; FOLKE, C.; BERKES, F.. Adaptive comanagement for building resilience in social-ecological systems. Environmental Management, v.34, n.1, p.75-90, 2004.

PACHECO, J. J.; PALHETA, M. V. O.; COSTA JUNIOR, R. N.; COSTA, S. V.; TOSTES, W. S.. Estatística municipal: Santarém. Belém, 2011.

R DEVELOPMENT CORE TEAM. R: A language and 
environment for statistical computing. Vienna: R Foundation for Statistical Computing, 2011.

RAGIN, C. C.. Qualitative Comparative Analysis Using Fuzzy Sets (fsQCA). In: Configurational Comparative Analysis. Sage Publications, 2007. p.87-121.

ROSAS, I. M. M. S.. Evaluación de la Resiliencia Socioecológica en Guanacaste, Costa Rica Casos: Nicoya, Hojancha y Nandayure. Centro Agronómico Tropical De Investigación Y Enseñanza, 2012.

ROSSETO, M.; HACKBART, R.. II Plano Nacional de Reforma Agrária: Paz, Produção e Qualidade de Vida no Meio Rural. Brasília, 2003.
SOUSA, E. S.. Uso integrado de dados de sensoriamento remoto para o estudo da geologia e geomorfologia da área da foz Rio Tapajós, Santarém, Pará. Manaus: Universiidade Federal do Amazonas, 2009.

VENTURIERI, A.; MONTEIRO, M. A.; MENEZES, C. R. C.. Zoneamento Ecológico-Econômico da Zona Oeste do Estado do Pará: diagnóstico socioambiental. Belém, 2010.

VICINI, L.; ADRIANO, O.; SOUZA, M.. Análise Multivariada Da Teoria À Prática. Santa Maria: UFSM, 2005.

WALKER, B.; HOLLING, C. S.; CARPENTER, S. R.; KINZIG, A. Resilience, Adaptability and Transformability in Socialecological Systems. Ecology and Society, v.9, n.2, p.5, 2004. 